\title{
Understanding Information Centric Layer of Adaptive Collaborative Caching Framework in Mobile Disconnection-Prone Networks
}

\author{
$\mathrm{Vu}$ San Ha Huynh \\ School of Computer Science \\ University of Nottingham \\ Nottingham, United Kingdom \\ vu.huynh@nottingham.ac.uk
}

\author{
Milena Radenkovic \\ School of Computer Science \\ University of Nottingham \\ Nottingham, United Kingdom \\ milena.radenkovic@nottingham.ac.uk
}

\author{
Robert John \\ School of Computer Science \\ University of Nottingham \\ Nottingham, United Kingdom \\ robert.john@nottingham.ac.uk
}

\begin{abstract}
Smart networks and services leverage in-network caching to improve transmission efficiency and support large amount of content sharing, decrease high operating costs and handle disconnections. In this paper, we investigate the complex challenges related to content popularity weighting process in collaborative caching algorithm in heterogeneous mobile disconnection prone environments. We describe a reputationbased popularity weighting mechanism built in informationcentric layer of our adaptive collaborative caching framework CafRepCache which considers a realistic case where caching points gathering content popularity observed by nodes differentiates between them according to node's reputation and network's connectivity. We extensively evaluate CafRepCache with competitive protocols across three heterogeneous real-world mobility, connectivity traces and use YouTube dataset for different workload and content popularity patterns. We show that our collaborative caching mechanism CafRepCache balances the trade-off that achieves higher cache hit ratio, efficiency and success ratios while keeping lower delays, packet loss and caching footprint compared to competing protocols across three traces in the face of dynamic mobility of publishers and subscribers.
\end{abstract}

Keywords- Opportunistic mobile networks, content discovery and retrieval, content caching, reputation, mobility and connectivity

\section{INTRODUCTION}

Current smart and cloud-based infrastructures, which are large-scale distributed network systems built on multi-layer architecture, are dealing with emerging challenges with respect to the dramatically increasing number of connected mobile devices and network traffic demands which involve distributed mobile decision making in real time. In dynamic mobile disconnection-prone networks, new technologies like the Fog Computing or the Internet of Things motivate the need to moving data and services from the core (e.g. centralised infrastructure/cloud services) to the edges of the network (e.g. people, vehicles, base stations, access points) with an aim to leverage information on the local available resources (energy, storage, computation), mobility of publishers/subscribers and dynamic content look up and notifications.

Powerful mobile devices allow an individual mobile device to be both cloud user and service provider. Efficiency, low-cost, timely manner and local relevance are the advantages of Mobile Edge/Fog Computing over conventional Internet Cloud. Current researches investigate complex challenges of the opportunistic discovery of content stored in remote mobile devices and the delivery to the requesting nodes in heterogeneous mobile disconnection prone environments. In our previous works, we proposed a latency-aware adaptive collaborative caching framework, CafRepCache [8], for content dissemination and query in heterogeneous mobile opportunistic networks and dynamic workloads. CafRepCache addresses complex open questions about decisions making in distributed opportunistic caching: where to cache and what to cache as well as how to manage the cache in order to maximise cache hit ratio while minimizing latency and avoiding network congestion. We built CafRepCache on dynamic predictive relative utilities and ensure all nodes in the network to be incentivised to collaborate.

In this paper, we focus on the information-centric layer of CafRepCache and investigate its collaborative cognitive caching framework by describing an integrated reputation aware weighting popularity mechanism in order to help caching points efficiently utilize the exchanged popularity values aggregated from other nodes through collaborative caching process, thus enhance the accuracy of its predictive content analytics and improve its cache hit ratio. Previous research has shown that collaborative caching usually outperforms locally optimized algorithms [20], thus node utility and content popularity are resolved by both local node and its neighbours. How to adaptively weight and combine the value of local observation and different collaborative observations is a challenging problem that need to be addressed in order to utilise efficiently the exchanged information, thus improve caching performance. We integrate "ego network" of each node as a dynamic network consisting of that node and contacts it meets most frequently or most recently. Ego network allows each node to give its own regional or temporal perspective of the network (or both are included). However, not every node's perspective of the network has the same value and level of accuracy. Thus, we describe our novel reputation-aware caching decision mechanism that evaluates and weighs different exchanged information derived from different nodes in the network based on node's reputation and connectivity, in order to improve the 
caching performance of our framework. Note that we assume there is no malicious behaviour from nodes in the network, thus focus on designing underlying algorithms that can adaptively utilise the exchanged resources from trusted collaborators.

The paper begins by providing an overview of the related work in section II, section III introduces reputation-aware collaborative adaptive caching CafRepCache model and scheme as well as describes its heuristics, section IV evaluates performance of different collaborative caching protocols associated with reputation-aware popularity weighting algorithms in opportunistic networks across a range of metrics over three heterogeneous realistic mobile social and vehicular traces for real-world workloads and content popularity from YouTube dataset. Section V gives a conclusion.

\section{RELATED WORK}

Authors in $[21,22,23]$ considered caching content at the edge of the mobile networks as the promising approach to address the network traffic demands from mobile users in which public or private transportation may act as mobile relays and caches. [21] and [22] then focus on the problem of downloading whole content and streaming videos from vehicles acting as mobile cloud. In our previous work [25], we envisaged a low cost mobile personal cloud architecture that is able to senses and adapts to the infrastructure behaviour, dynamic social ties, reputation of other nodes and uses intelligent network communication for increased reliability. In [23], authors assumed that content is streamed chunk-by-chunk and if a chunk can be downloaded from an encountered vehicle before it must be played, this is data traffic that is offloaded from the main infrastructure or expensive macro cell links without any visible impact on the use.

Authors in [19] studied the effect of cooperative caching schemes on delay tolerant mobile network. [19] designed a mathematical model of mobile content sharing network based on file popularity distribution, user mobility and delay tolerance. Authors in [14] combine betweenness, similarity and tie strength for social routing metric which directs the traffic to more central nodes, and thus increases the probability of finding the optimal relay for delivering packets but congests the points that have higher social centrality. [4] proposed Café and CafeREP, a congestion-aware mobile social framework for data forwarding over heterogeneous opportunistic networks. Our previous work [8] describes our early proof of concept new latency aware collaborative cognitive caching approach in heterogeneous opportunistic mobile networks which utilises fully localised and ego networks multi-layer predictive heuristics about dynamically changing topology, resources and popularity content. In this paper, we base our work on $[4,8]$ and focus on resolving the content popularity based on reputation through collaborative caching process.

[18] proposed a reputation framework based on mobility and centrality to improve security in highly mobile, sparse and disconnected environments. Authors integrated centrality, local observations, selective deviation test and adaptive timer techniques in the reputation model to improve efficiently node monitoring and trust resolution. [3] proposed a reputation aware adaptive obfuscation in mobile opportunistic networks based on implicit reputation building, complex graph connectivity analytics and obfuscation history analyses. The authors presented testing scheme and hierarchical reputation states to enable an accurate, intelligent and efficient fully distributed reputation building based on the nodes' obfuscation behaviour. Authors in [24] proposed an intelligent cooperative sensing framework that enhances the reliability of the manufacturing plant in the disconnection prone heterogeneous sensor networks. Each node has its own trust value which is resolved based on the weighted sum of multiple nodes' observations of the node. The framework identifies and uses nodes that have both high centrality and reputation to improve the reliability and accelerate reputation convergence while being able to detect and isolate faulty nodes that have low trust value. In [5], authors propose fully distributed reputation mechanism for next generation mobile offloading in resource constrained mobile networks. [7] presented a Fuzzy-based qualified voting model in which every peer has a voting score according to its behaviours and the success or failure of its actions. The authors stated that Fuzzy Logic helps to deal with imprecision and approximate reasoning that are involved in the given problem [6]. Thus, the proposed system could choose reliable peers with good voting score to make collaborative decisions in P2P mobile environment. In this paper, we compare our reputation aware collaborative caching against Fuzzy-based qualified voting model in [7]. We follows distributed reputation guidelines given in $[2,9,10]$, considers sociality metrics [14] and reputation testing in $[3,18]$ to propose a reputation-based popularity weighting mechanism built in information-centric layer of the collaborative adaptive caching framework CafRepCache [8] in order to improve the accuracy of its predictive content analytics.

\section{ADAPTIVE COLLABORATIVE CACHING FRAMEWORK}

Our adaptive collaborative caching framework CafRepCache has a distributed cross-layer structure as shown in [8]. Reputation system built in the content layer assisting the caching cooperation process by adaptively weighting popularity values aggregated from other nodes. We first describe briefly the system model and related assumptions used in CafRepCache framework [8], then we focus on our reputation building architecture of the content layer and its functional overview.

\section{A. System Model}

We model a system as a network $G$ that consists of a set $N$ of nodes and a set $E$ of edges, $G=(N, E)$. As the connectivity of the network and the state of the nodes change over time, we model each of these sets as time series, thus $N=\left\{N^{t}: \mathrm{t} \in \mathrm{T}\right\}$ and $E=\left\{E^{t}: \mathrm{t} \in \mathrm{T}\right\}$.

We assume that each node in the network $n_{i} \in N$ has a cache of size $\theta_{i}$. We denote with $O$ a set of content files that can be requested by the network. Each content $o_{k}^{t} \in O$ (or $o_{k}$ for simplicity) is published at time $t$ and has the size $\delta_{k}$. At each node $n_{i} \in N, q_{i, k}^{t}$ is the normalised request rate of the content $o_{k}$ (i.e. content popularity) observed locally from $n_{i}$ at time $t$, $\sum_{k} q_{i, k}^{t}=1 ; z_{i, k}^{t}$ is the normalised aggregated request rate of the content $o_{k}$ observed from all the neighbours of $n_{i}$ at time $t$, $\sum_{k} z_{i, k}^{t}=1$. When two nodes are in contact, they exchange their local content popularity observation. Each node continuously resolves the value of dynamically changing content popularity 
based on its local observation and the collaborative observations it gets from others. $W\left(q_{i, k}^{t}, z_{i, k}^{t}\right)=\alpha q_{i, k}^{t}+\beta z_{i, k}^{t}$ denotes the function to weight the value of collaborative observations over local observation.

We integrate "ego network" of each node $n_{i}: E N_{i}$ as a dynamic network consisting of a the node $n_{i}$ and contacts it meets most frequently or most recently. In this way, ego network allows each node to give its own regional or temporal perspective of the network (or both are included).

Each node $n_{i}$ has a node utility value which consists of social utility (betweenness, similarity, tie strength), resource utility (retentiveness, receptiveness, congesting rate), energy utility and end-user utility.

\section{B. Reputation-Based Content Popularity Weighting Mechanism}

Our distributed reputation aware mechanism built within information-centric layer of CafRepCache integrates four main features: centrality, credit-based feedback, reputation discounting and secondary response (as in line with $[1,2,18]$ ) to weigh the value of exchanged content popularities among ego network nodes in order to improve the collaborative caching decisions in highly mobile and disconnection-prone scenarios. Fig. 1 shows the interaction between the key components of our reputation model in order to provide automatic and autonomous weighing collaborative content popularities based on the available neighbours' reputations.

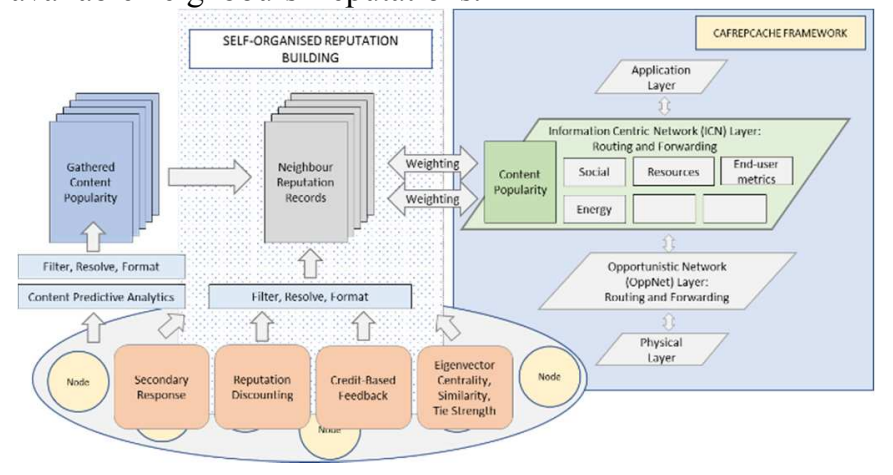

Figure 1. Architecture \& Reputation system model.

Given a caching point $n_{i}$ has a request to resolve a popularity of a certain content $o_{k}$ in order to make a caching decision (e.g. whether to cache that content or not, whether to choose to delegate or remove the content, etc.). Through the collaborative caching process, $n_{i}$ aggregates different popularity value $p_{j, k}$ of content $o_{k}$ observed from each neighbor node $n_{j} \in E N_{i}$ in its ego network $E N_{i}$. Assuming that $n_{i}$ take account equal for every node, the aggregated content popularity of the content $o_{k}$ observed from all the neighbours of $n_{i}$ is:

$$
z_{i, k}=\frac{1}{\left|E N_{i}\right|} \sum_{n_{j} \in E N_{i}} p_{j, k}
$$

However, we argue that not every node's perspective of the network has the same value and level of accuracy, thus we weigh the value of content popularity observed from a node based on its reputation. Firstly, we measure a node's reputation based on its centrality, similarity and tie strength $[9,14]$, to the caching point $n_{i}$.

Nodes with higher centrality have higher connectivity or higher probability of getting in contact with many other nodes than nodes with lower centrality, thus its observation on a popularity of a particular content is preferred. Let $A_{i, j}$ be the adjacency matrix, i.e. $A_{i, j}=1$ if $n_{i}$ is linked to $n_{j}$ and $A_{i, j}=0$ otherwise. The relative eigenvector centrality $[2,10]$ of $n_{i}$ is defined as:

$$
x_{i}=\frac{1}{\lambda} \sum_{n_{j} \in E N_{i}} x_{j}=\frac{1}{\lambda} \sum_{j=1}^{N} A_{i, j} x_{j}
$$

in which $\lambda$ is a constant.

Moreover, we argue that nodes with higher similarity value $[9,14]$ with the caching point may help to give a short-term predictive content popularity due to the spatial and temporal locality of content requests. The similarity degree between $n_{i}$ and $n_{j}$ is measured by $\left|N_{i} \cap N_{j}\right|$ as the similarity of contact between node $n_{i}$ and $n_{j}$

On the other hand, we still consider nodes which have weak tie strength [14] with the caching point as we argue that these nodes may have a completely different connectivity patterns compared to the caching point, thus may give a wider and broaden long-term predictive content popularity. The tie strength value between node $n_{i}$ and $n_{j}$ is defined as:

$$
T S=\frac{f(j)}{F(i)-f(j)}+\frac{r e c(j)}{T(i)-r e c(j)}+\frac{d(j)}{D(i)-d(j)}
$$

Through our experiments, we observed that centrality and similarity have a major contribution to the reputation building due to the spatial and temporal locality of content request whiles nodes with weak tie strength does not add significant value to the accuracy of aggregated content popularity. Assuming that $n_{i}$ take social metrics as the first reputation into account for every node, the aggregated content popularity of the content $o_{k}$ observed from all the neighbours of $n_{i}$ is now rewritten as:

$$
z_{i, k}=\frac{1}{\left|E N_{i}\right|} \sum_{n_{j} \in E N_{i}} \text { SocialDegre }_{i, j} \cdot p_{j, k}
$$

SocialDegree $_{i, j}=\{$ Centrality, Similarity, Tie Strength $\}$

Then, we base on our previous work $[1,3,18]$ to propose credit-based feedback algorithm in order to evaluate the accuracy and usefulness the content popularity value reported from a node in ego networks to a caching point.

At the beginning, we assume that the reputation of all nodes in the network is equal to their social degree as described above. After the first exchanging information, node $n_{i}$ gets the collaborative content popularity from others and weight them based on its reputation. After the first cache hit, caching point $n_{i}$ weights up the reputation value of nodes who reported correctly and weights down the reputation value of those who reported incorrectly.

$$
\left\{\begin{array}{c}
R_{i, j}(t)=\text { SocialDegree }_{i, j} \text { when } t=0 \\
R_{i, j}(t)=R_{i, j}(t-1) \pm \partial
\end{array}\right.
$$

where $\partial$ is the value added or deducted based on the success or failure of content popularity prediction of a collaborative node. $\partial$ value is proportional to the gap between the content 
popularity value it reported to $n_{i}$ and the actual content popularity $n_{i}$ experiences after a certain time window $\Delta \mathrm{T}$.

The reputation of a node decreases when it reported to $n_{i}$ that a certain content is high popular but $n_{i}$ does not encounter any request for that content, and vice versa. In this case, $\partial$ value added or deducted is proportional to the time duration $\Delta \mathrm{T}$.

In addition to using historical observations, we also use adaptive expiration timer, reputation discounting and secondary response $[1,2,18]$ specifically tailored for our highly challenged environment in order to increase the accuracy and reliability of the reputation resolution. Each node holds $\mathrm{N}$ neighbour reputation records where $\mathrm{N}$ can be determined by the node's resource capacity. Nodes with higher reputation and centrality should hold enough reputation records about other nodes in order to provide sufficient coverage of the nodes in its own area. Node recycles these records using expiration time to balance the trade-off between different overheads and the need to have enough reputation about different neighbours. Our protocol uses reputation discounting to ensure that old reputations will age giving more chance for nodes to reclaim their reputation by consistently behaving in a cooperative manner and contributing to the aggregated content popularity. The reputation value of a node ages along time that:

$R_{i, j}(t)=\alpha * R_{i, j}(t-1)$ where $\alpha$ is aging factor.

The secondary response avoids reputation discounting backfires by increasing sensitivity to nodes that have been deemed predictively inaccurate in the past.

Assuming that $n_{i}$ take reputation into account for every node, the aggregated content popularity of the content $o_{k}$ observed from all the neighbours of $n_{i}$ is now rewritten as:

$$
z_{i, k}=\frac{1}{\left|E N_{i}\right|} \sum_{n_{j} \in E N_{i}} R_{i, j} p_{j, k}
$$

\section{EVALUATION}

In this section, we present and evaluate the performance of CafRepCache against state-of-the-art caching protocols including LFU and Fuzzy Cache [7] while varying the number of contents and the cache buffer size. In our experiments, we tailor LFU to not only keep a counter associated each content as popularity value but also to collaboratively exchange the counter value between its neighbours. The aggregated popularity value is resolved by equally weighting all content popularity values gathered from others. Fuzzy Cache [7] uses the Fuzzy Logic based-qualified voting model to weight the exchanged content popularity which in turn is resolved by the number of requests. In order to enable fair analysis, we implement LFU and Fuzzy Cache over social forwarding as other algorithms have different social forwarding algorithms and all three traces have been shown to have social character.

As mobility and connectivity of the nodes have a major impact on the performance of any opportunistic communication protocol, it is fundamental to evaluate our caching algorithm over multiple heterogeneous real-world mobile data sets. We use San Francisco Cab [15], RollerNet [16] and Infocom [17] traces in ONE [13]. San Francisco Cab Trace [15] includes GPS traces of 550 cabs over a period of 30 days in the San Francisco Bay Area. RollerNet [16] spans three hours during which 62 roller- bladders travel about 20 miles in Paris and utilize Bluetooth on their cell phones for communication. Infocom [17] is a 4-day trace that consists of 78 volunteers equipped with Bluetooth devices and additional 20 static long-range devices placed at various semi-static and static locations of the conference venue.

San Francisco trace [15] is the most challenging trace compared with RollerNet [16] and Infocom [17] due to very short connectivity durations, very high disconnections and low number of contacts during connected times. We observe that both San Francisco and RollerNet traces exhibit short contact durations (a mean of $45 \mathrm{~s}$ and $33 \mathrm{~s}$, a median of $11 \mathrm{~s}$ and $24 \mathrm{~s}$ and a maximum of $73 \mathrm{~s}$ and $42 \mathrm{~s}$ respectively) while Infocom has substantially longer contact durations (a mean of $2.5 \mathrm{~min}$, a median of $2 \mathrm{~min}$ and a maximum value of $4 \mathrm{~min}$ ). San Francisco trace suffers from the longest isolation periods (a mean of $0.5 \mathrm{~h}$, a median of $1.7 \mathrm{~h}$ and a maximum value of $3 \mathrm{~h}$ ) compared to RollerNet and Infocom (with a mean of $1.5 \mathrm{~min}$ and $4 \mathrm{~min}$, a median of $1 \mathrm{~min}$ and $6 \mathrm{~min}$, and a maximum of $4 \mathrm{~min}$ and 10 min respectively). In addition, RollerNet trace has the highest observed number of contacts compared to San Francisco Cab and Infocom traces.

We use YouTube Dataset [11,12] as a real trace for content requests. The dataset contains total 2135798 videos, each has video id, length, views and other attributes. Based on the assumption that file size is proportional to the video length, we set up the average file size is $8.4 \mathrm{MB}$, which is in line with $[11,12]$. We also assume that contents are uniformly distributed among publishers. All caching points in the network have a uniform storage capacity.

In the first set of experiments, we compare CafRepCache against state-of-the-art caching protocols LFU and Fuzzy Cache [7] associated with different content-popularity weighting algorithms in opportunistic networks over different criteria: cache hit ratio, success ratio, delay and packet loss in the face of dynamically varying number of total content in order to evaluate our collaborative caching algorithm in the presence of dynamic content population. Due to space limitation, only the cache hit ratio will be shown.

In the second set of experiments, we conduct performance analysis of CafRepCache against LFU and Fuzzy Cache while dynamically varying cache buffer size. We show that CafRepCache outperforms other competing protocols in terms of cache hit ratio, success ratio, delay, packet loss and relative footprint reduction for the end to end content retrieval process in the presence of heterogeneous buffer size.

\section{A. Evaluation in the presence of dynamic content population}

We run six increments with number of contents ranging from 10 to $10^{6}$ contents. For simplicity and to provide sensitivity analysis of content size and buffer capacity, we limit the upper-bound of number of content to $10^{6}$ without losing generality. We also assume that contents are uniformly distributed among publishers.

All caching points in the network have a uniform storage capacity such that the total caching space could fit at $0.1 \%$ of the total contents population. For each experiment with different content population, we assume that a random $50 \%$ of node population are subscribers and a random $25 \%$ of nodes are 
publishers; subscribers and publishers are mobile and not uniformly distributed as well as that they can have different connectivity patterns. All experiments are repeated ten times and averaged.

We begin by analysing performance of cache hit ratio which refers to how many interest packets are matched with the contents in caching points without being forwarded to the caching decision-making later on. Thus, multi-layer realtime predictive heuristics allows CafRepCache to manage complex dynamic trade-offs between dynamically changing topology, dynamic resources and varying content popularity and interest. CafRepCache is followed by Fuzzy Cache and LFU range from $27 \%-55 \%$ for all traces with respect to the increase of content population. We observe that Fuzzy Cache
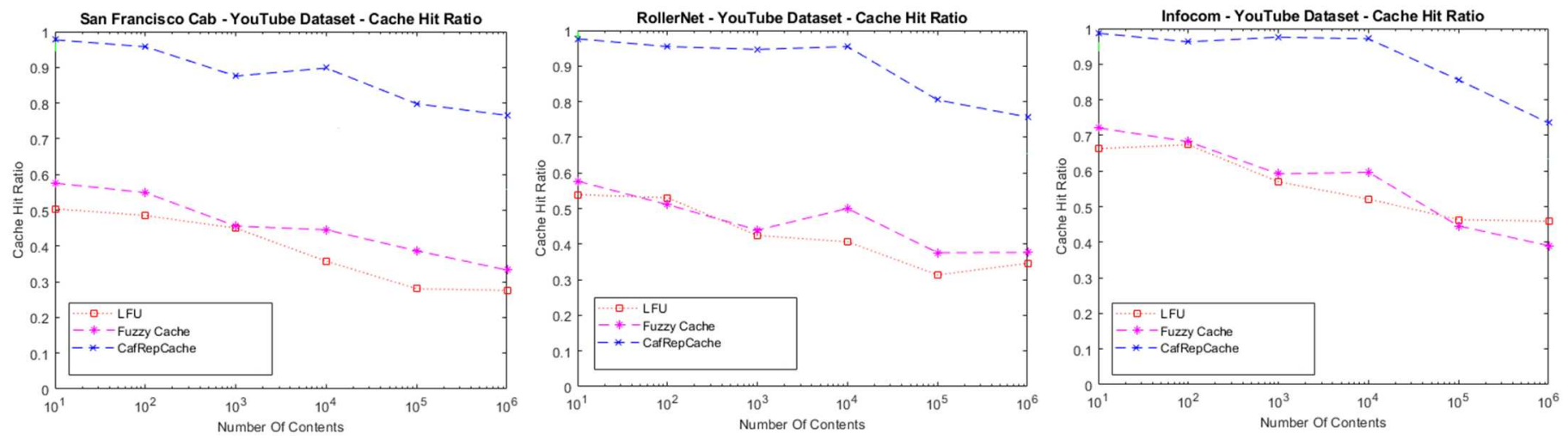

Figure 2. Cache Hit Ratio vs. Number of Contents

publishers and indicates efficiency of caching decisions and locations. In Fig. 2, we show that CafRepCache achieves the highest cache hit ratio (typically around $90 \%$ for all three traces and in the face of dynamic content population) compared to state of the art algorithms LFU and Fuzzy Cache. When the number of contents increases, CafRepCache still keep above $80 \%$ cache hit ratio for all three traces. This is because CafRepCache profits from adaptive caching and more accurate content popularity prediction. CafRepCache is able to take advantage of highly skewed content popularity and content request locality to efficiently predict the incoming content requests based on its local observation and collaborative observations from the others. It profits from favouring some nodes' exchanged popularity value based on their sociality degree, assuming that nodes with higher centrality have higher connectivity or higher probability of getting in contact with many other nodes than nodes with lower centrality, thus its observation on a popularity of a particular content is preferred. Moreover, the credit-based feedback module allows CafRepCache to adaptively weight and utilize efficiently the exchanged popularity values obtained from other nodes within its ego network over time, upgrade or downgrade some nodes' observations based on the accuracy and usefulness they offer to has no (or little) improvement to the accuracy of predicting content popularity compared to the traditional caching algorithm LFU.

\section{B. Evaluation in the presence of dynamic cache buffer size}

In order to evaluate the accuracy of CafRepCache, Fuzzy Cache and LFU in predicting content popularity, we first limit and then increase the cache buffer size while evaluating cache hit ratio, success ratio, delay and packet loss of these weighting popularity algorithms. Due to space limitation, only the cache hit ratio will be shown in this paper.

Smaller cache buffer size offers a more selective cached contents, thus requires more accurate content popularity prediction in order to minimise the cache miss ratio. In Fig. 3, we show that CafRepCache achieves the highest cache hit ratio (above $70 \%$ when the cache space is very limited and typically around $92 \%$ for all three traces when more cache space is given) compared to state of the art algorithms. This is because the reputation-based popularity weighting module built on information-centric layer of CafRepCache improves the predictive content analytics, allows it to weigh efficiently the collaborative content popularity reported from the others based on their sociality and centrality while being able to adaptively
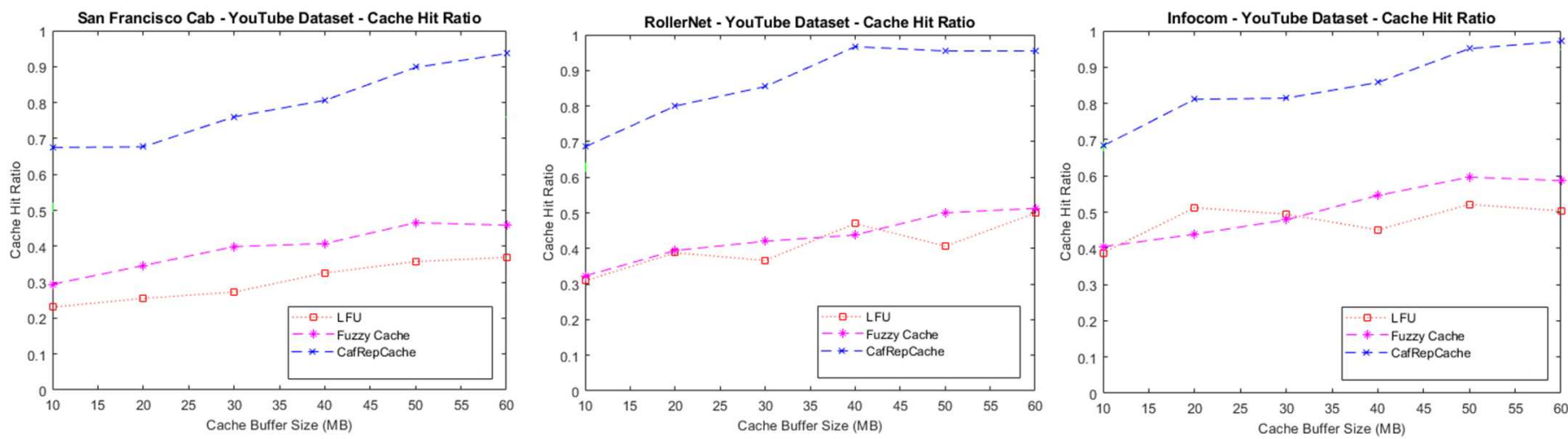

Figure 3. Cache Hit Ratio vs. Cache Buffer Size 
test, discount or recover nodes' reputation over time. More accurate aggregated content popularity leads to more accurate final content popularity measurement, thus helps caching points to efficiently predict the incoming content requests and improve its cache hit ratio.

Fig. 3 shows that equal popularity weighing will not be sufficient to optimally weight others' observations as it shows that CafRepCache is followed by LFU and Fuzzy Cache (range from 22\%-47\%). Fuzzy Cache and LFU performance are relatively similar, Fuzzy Cache [7] with Fuzzy Logic basedqualified voting model relies heavily on a static set of rules and knowledge base, thus may not be able to adaptively weight the node's reputation over time with respect to the dynamic changing of nodes' mobility, connectivity, resources and workload. In fact, Fig. 3 shows that Fuzzy Cache makes no significant improvement compared to traditional caching algorithm LFU.

\section{CONCLUSION}

This paper described collaborative adaptive caching framework CafRepCache [8] with reputation-based popularity weighing mechanism built in information-centric layer of CafRepCache to adaptively weight different exchanged content popularity values gathered from different nodes in the ego network based on node's reputation and connectivity, in order to improve the predictive caching performance. We show that CafRepCache significantly improves the performance of predictive caching over very different time-varying real world network topologies, mobility patterns and content requests compared to other state-of-the-art weighting popularity algorithms. Reputation-based popularity weigting mechanism built in CafRepCache allows it to take advantage of highly skewed content popularity and content request locality using sociality metrics while being able to test, discount and recover the reputation over time in order to utilize efficiently the exchanged popularity values obtained from other nodes, thus improve significantly the predictive caching decisions. For our future work, we plan to investigate CafRepCache caching framework in chunk-level object popularity that leverages the sequential correlation between chunk requests of the same file in order to improve the cache performance. We also plan to exploit the energy efficient data sharing approaches that will make CafRepCache smart data dissemination and query more usable, reliable and scalable in opportunistic disconnection tolerant networks.

\section{REFERENCES}

[1] M. Radenkovic, A. Benslimane, and D. McAuley. Reputation Aware Obfuscation for Mobile Opportunistic Networks. IEEE Transactions on Parallel and Distributed Systems, vol. 26, no. 1, pp. 230-240, 2015.

[2] S. Buchegger. Reputation Systems for Self-Organized Networks: Lessons Learned. IEEE Technology and Society Magazine, Toward Fourth Generation Wireless, p. 1-10. 2008

[3] M. Radenkovic, I. Vaghi, Adaptive User Anonymity for Mobile Opportunistic Networks. Proceedings of ACM Mobicom CHANTS, Istanbul, Turkey, August, p. 79-82, 2012.
[4] M. Radenkovic, A. Grundy. Efficient and Adaptive Congestion Control for Heterogeneous Delay Tolerant Networks. Ad Hoc Networks, v. 10, n. 7, 2012.

[5] H. Flores, R. Sharma, D. Ferreira, V. Kostakos, J. Manner, S. Tarkoma, P. Hui, Yong L. Social-aware Hybrid Mobile Offloading. Pervasive and Mobile Computing 36, p.25-43, 2017.

[6] L. Zadeh. Fuzzy Logic, Neural Networks, and Soft Computing. ACM Communications, p. 77-84, 1994.

[7] Y. Liu, S. Sakamoto, L. Barolli, M. Ikeda. A Fuzzy-based System for Qualified Voting in P2P Mobile Collaborative Team: Effects of Member Activity Failure. IEEE 31st International Conference on Advanced Information Networking and Applications, p. 639-64, Taipei, 2017.

[8] M. Radenkovic and V.S.H. Huynh,. Collaborative Cognitive Content Dissemination and Query in Heterogeneous Mobile Opportunistic Networks. ACM SMARTOBJECTS 2017, Mobicom USA, p. 7- 12, 2017.

[9] E. Daly. Harnessing wisdom of the crowds dynamics for time-dependent reputation and ranking. Proc. IEEE Int. Conf. Adv. Social Netw. Anal. 913 Mining, Athens, Greece, 2009, pp. 267-272.

[10] S. Buchegger, J. Mundinger, Jean-Yves Le Boudec. Reputation Systems for Self-Organized Networks. IEEE Technol. Soc. Mag. 27(1) p. 41-47. 2008 .

[11] M. Cha, H. Kwak, P. Rodriguez, Y.-Y. Ahn, and S. Moon. I tube, you tube, everybody tubes: Analyzing the world's largest user generated content video system. Proc. ACM IMC, Oct. 2007.

[12] Xu Cheng, C. Dale, and Jiangchuan Liu. Statistics and social network of youtube videos. 16th Int. Workshop on Quality of Service (IWQoS), p. 229-238. 2008.

[13] A. Keränen, J. Ott and T. Kärkkäinen. The ONE Simulator for DTN Protocol Evaluation. SIMUTools'09: 2nd International Conference on Simulation Tools and Techniques. Rome. 2009.

[14] E. Daly and M. Haahr. Social network analysis for routing in disconnected delay-tolerant manets. Proceedings of ACM MobiHoc, 2007.

[15] San Francisco cabs data traces, downloaded from the Cab Spotting project, $<$ http://cabspotting.org/api $>$ for the period 20 Sep 2011- 20 Oct 2011.

[16] J Leguay, F Benbadis. CRAWDAD data set upmc/rollernet (v. 2009-0202), Feb, 2009.

[17] Scott, R. Gass, J. Crowcroft, P. Hui, C. Diot, A. Chaintreau. CRAWDAD data set Cambridge/haggle (v. 2009-05-29), May 2009.

[18] Zakhary, S.R., Radenkovic, M. Reputation-based security protocol for MANETs in highly mobile disconnection-prone environments. Proc. IEEE/IFIP WONS 2010, p. 161- 167. 2010.

[19] T. Wang, P. Hui, S. R. Kulkarni, and P. Cuff. Cooperative Caching based on File Popularity Ranking in Delay Tolerant Networks. Proc. ExtremeCom, Zurich, " Switzerland, March 2012.

[20] D. Bertsimas, V. F. Farias and N. Trichakis. The price of fairness. Operations Research. v. 59, n. 1, 2011.

[21] L. Vigneri, T. Spyropoulos, C. Barakat. Storage on Wheels: Offloading Popular Contents through a Vehicular Cloud. IEEE 17th International Symposium on A World of Wireless, Mobile and Multimedia Networks (WoWMoM) 2016.

[22] L. Vigneri, T. Spyropoulos, C Barakat. Streaming Content from a Vehicular Cloud. Proceedings of ACM Mobicom CHANTS '16, New York, USA, p. 39-44. 2016.

[23] L. Vigneri, S Pecoraro, T. Spyropoulos, C. Barakat. Per-Chunk Caching for Video Streaming from a Vehicular Cloud. In ACM Mobicom CHANTS '17 p.57-62. USA 2017.

[24] M. Radenkovic, I. Kostadinov and B. Wietrzyk. Increasing Communication Reliability in Manufacturing Environments. Proc. IEEE IWCMC, Croatia. p. 1377-1383, 2015.

[25] M. Radenkovic. Cognitive Privacy for Personal Clouds. Cognition and Cooperation in Advanced Wireless and Mobile Information Systems, Mobile Information Systems. 2016(1): 31. 2016. 